Check for updates

Cite this: RSC Adv., 2018, 8, 2280

Received 3rd October 2017

Accepted 5th December 2017

DOI: $10.1039 / c 7 r a 10906 g$

rsc.li/rsc-advances

\section{Neuroprotective effect of arctigenin against neuroinflammation and oxidative stress induced by rotenone}

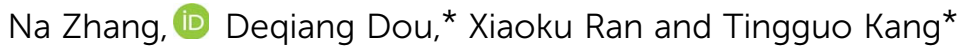

Background: the present study was to investigate the neuroprotective effect of arctigenin, the major active component of a traditional Chinese medicine "Arctii Fructus", against PD in a rat model induced by rotenone. Materials and methods: in the present study, rotenone was injected subcutaneously in the backs of rats to mimic the progressive neurodegenerative nature of PD and arctigenin was administered. Behavioral analyses including a grid test, bar test and open-field test were used to evaluate motor activities and behavioral movement abilities. Energy metabolism indexes including oxygen consumption, carbon dioxide production, heat production and energy expenditure were measured via a TSE phenoMaster/LabMaster animal monitoring system. Immunohistochemistry was performed to detect the staining of $\mathrm{TH}$ and the expression of $\alpha$-synuclein in substantia nigra (SN). The effect of arctigenin on oxidative stress was evaluated by the levels of GSH and MDA, and activities of SOD and GSH-Px. The levels of pro-inflammatory cytokines such as IL-6, IL-1 $\beta$, TNF- $\alpha$, IFN- $\gamma$ and PGE2, the expression of Iba-1 and GFAP, and the impression of inflammatory mediators such as COX-2 and NF- $\kappa B$ in the SN were measured to evaluate the effect on the inflammation of $\mathrm{SN}$ area induced by rotenone. Results: compared with the ROT group, the deadlock time of rats treated with arctigenin was significantly shortened and the score of locomotor activity increased in the behavioral test; the number of $\mathrm{TH}^{+}$ positive DA neurons of the arctigenin treated group was increased and $\alpha$-synuclein immunopositive was decreased; the level of GSH and activities of SOD and GSH-Px in the arctigenin-treated group were significantly increased; arctigenin administration induced a significant decrease in the MDA level; arctigenin also significantly decreased the levels of IL- $6, I L-1 \beta, T N F-\alpha, I F N-\gamma$ and PGE2 and reduced the impression of COX-2 and NF- $\mathrm{B}$ in $\mathrm{SN}$; treatment with arctigenin decreased microglia and astrocyte activation evidenced by the reduced expression of Iba-1 and GFAP. Conclusion: the findings demonstrated that arctigenin can improve the behavior changes of PD rats and the damage of DA neurons. The oxidative stress and inflammation involved in the pathogenesis of PD and arctigenin may protect DA neurons through its potent antioxidant and anti-inflammatory activities.
\end{abstract}

\section{Introduction}

Parkinson disease (PD), the second most common neurodegenerative disease after Alzheimer's disease, is pathologically characterized by the progressive loss of dopaminergic neurons in the substantia nigra pars compacta $(\mathrm{SNc})$ and the presence of Lewy bodies following gradual dysfunction of the basal ganglia in the central nervous system. ${ }^{1}$ As a result, the level of dopamine (DA) is significantly reduced in the striatum. The clinical symptoms of PD include impairments in movement, resting tremor, bradykinesia, muscular rigidity, postural instability, and disturbance in gait, attributable to dopaminergic neurodegeneration. The increased reactive oxygen species (ROS)

College of Pharmacy, Liaoning University of Traditional Chinese Medicine, 77 Life One Road, DD Port, Dalian 116600, PR China. E-mail: kangtg@lnutcm.edu.cn; deqiangdou@126.com generated from multiple sources, such as dopamine metabolism, mitochondrial dysfunction, altered iron homeostasis, neuroinflammation, and aging, result in progressive and chronic features. Recently, numerous studies reported the involvement of several pathological mechanisms responsible for the loss of dopaminergic neurons in PD. Although the molecular mechanisms of neuronal cell death in PD is not completely understood, oxidative stress and neuroinflammation appear to play key roles in both initiation and progression of $\mathrm{PD}^{2,3}$

Rotenone, a natural organic pesticide in agriculture, is an inhibitor of mitochondrial electron transfer chain complex I. ${ }^{4}$ Owing to its lipophilic property, the complex I inhibitor is easy to pass through the blood-brain barrier and cause selective dopaminergic degeneration., ${ }^{5,6}$ Rotenone-treated animals represent pathological features that are similar to those observed in human PD patients such as the progressive loss of 
DA neurons in SNc, $\alpha$-synuclein aggregation in DA neurons, formation of Lewy body, oxidative stress, mitochondrial impairment, microglial activation and neuroinflammation. ${ }^{7-10}$ Therefore, the rotenone-induced rat induced a PD-like phenotype for understanding PD pathogenesis and investigating novel therapeutic agents in PD. ${ }^{\mathbf{1 1}}$

The current available therapies for PD mainly focus on improving the symptoms and delay or prevent the progression of the disease. ${ }^{12}$ Additionally, motor complications and the adverse effects of the available drugs remain huge challenges for long-term therapy. ${ }^{13}$ Numerous experimental studies demonstrated that compounds which had antioxidant and antiinflammatory activity are neuroprotective and may be a promising disease modifier for PD. ${ }^{14-16}$ There was still considerable interest in the development of neuroprotective drugs from natural origins as a therapeutic strategy for PD. ${ }^{17}$

Arctigenin, extracted from Arctii Fructus, appears promising for its therapeutic potential including anti-inflammation, antioxidation and neuron protective activities. ${ }^{18-22}$ In addition, it has been reported that arctigenin can be used to treat neurodegenerative diseases such as Alzheimer's disease (AD). The result showed that the treatment of arctigenin in mice highly decreased $A \beta$ formation and senile plaques and efficiently ameliorated $\mathrm{AD}$ mouse memory impairment, strongly highlighting the potential of arctigenin in anti-AD drug discovery. ${ }^{23}$ Therefore, the aim of the present study was to investigate the potential preventive and therapeutic effects of arctigenin from anti-oxidative stress and anti-inflammatory point of view in rotenone-induced $\mathrm{PD}$, an in vivo rat model which mimics most of the pathological features of human PD characterized by loss of dopaminergic neurons, formation of Lewy body-like inclusions. ${ }^{11}$

\section{Materials and methods}

\subsection{Drugs and chemicals}

Arctigenin was extracted from Fructus arctii, and the purity of arctigenin was determined to be $99 \%$ by high-performance liquid chromatography. Rotenone and dimethylsulfoxide (DMSO) were purchased from Sigma-Aldrich. The antibodies of nuclear factor $\kappa \mathrm{B}(\mathrm{NF}-\kappa \mathrm{B})$ and cyclooxygenase-2 (COX-2) were from Abcom. The antibodies of ionized calcium binding adaptor molecule-1 (Iba-1), glial fibrillary acidic protein (GFAP) and tyrosine hydroxylase (TH) were from BOSTER Biological Technology Co. Ltd. The antibodies of $\alpha$-synuclein was from ZSGB-BIO. Enzyme linked immunosorbent assay (ELISA) kits for DA, interleukin 6 (IL-6), interleukin $1 \beta$ (IL-1 $\beta$ ), tumor necrosis factor- $\alpha$ (TNF- $\alpha$ ), interferon- $\gamma$ (IFN- $\gamma$ ), and prostaglandin E2 (PGE2) and the assay kits for superoxide dismutase (SOD), glutathione (GSH), glutathione peroxidase (GSH-Px) and malonaldehyde (MDA) were purchased from Nanjing Jiancheng Bioengineering Institute.

\subsection{Experimental animals}

SD male rat (180-220 g) were purchased from Liaoning Chang Sheng Biotechnology Co. LTD [license: SCXK (Liao) 2010-0001].
All rats were maintained with free access to food and water in plastic cages at $22 \pm 2{ }^{\circ} \mathrm{C}$, a relative humidity of $50-60 \%$, and kept on a $12 \mathrm{~h}$ light/dark cycle. Animals were housed for one week prior to the experiments. All animal procedures were performed in accordance with the Guidelines for Care and Use of Laboratory Animals of Liaoning University of Traditional Chinese Medicine (131/2010) and approved by the Animal Ethics Committee of Liaoning Provincial Animal Welfare and Care Guidelines.

\subsection{Experimental design}

The SD rats were randomly divided into six groups $(n=20$ per group): group I, vehicle-injected control group (CON), group II, rotenone-injected and vehicle-treated group (ROT), group III, rotenone-injected and $50 \mathrm{mg} \mathrm{kg} \mathrm{kg}^{-1}$ Madopa-treated group (MDP), group IV, rotenone-injected and $20 \mathrm{mg} \mathrm{kg}^{-1}$ arctigenintreated group (LAG), group V, rotenone-injected and $60 \mathrm{mg} \mathrm{kg}$ arctigenin-treated group (MAG), and group VI, rotenoneinjected and $150 \mathrm{mg} \mathrm{kg} \mathrm{kg}^{-1}$ arctigenin-treated group (HAG). Rotenone was first dissolved in DMSO at $50 \times$ stock solution and diluted in sunflower oil to obtain a final concentration of $2.0 \mathrm{mg} \mathrm{mL}^{-1}$. For the induction of PD in rats, rotenone $(2.0 \mathrm{mg}$ per $\mathrm{kg}$ body weight) was administered intraperitoneally (i.p.) once a day for 5 weeks. To test the neuroprotective efficacy of arctigenin, it was diluted in $20 \%$ polyethylene glycol 400 and administered intragastrically (i.g.) at dose of 20, 60 and $150 \mathrm{mg} \mathrm{kg} \mathrm{kg}^{-1}$ once daily for 5 weeks, $60 \mathrm{~min}$ prior to ROT injection. The CON and ROT groups received the similar amount of vehicle only.

\subsection{Behavioral test}

The open field test was applied to evaluate the behaviors of rats. ${ }^{24}$ The open field arena sized $100 \mathrm{~cm} \times 100 \mathrm{~cm} \times 50 \mathrm{~cm}$ was made of plastic box and the floor was painted with white lines that formed $25 \times 25 \mathrm{~cm}$ patterns. The rats were placed individually in the central area of the open field system and its behavior was recorded for $3 \mathrm{~min}$. The observed indicators included the followings: (1) the number of boxes that the rats moved, and (2) the duration in which the forelimbs were off the ground by more than $1 \mathrm{~cm}$. Additionally, gait instability, muscle tremors, slowed activity and other abnormal behaviors were also observed. Between each session, the apparatus was thoroughly cleaned with alcohol and dried.

For the pole test,$^{25}$ the forepaws of rats were placed on a bar which was $9 \mathrm{~cm}$ above the base. The end point of pole test is considered when the front paws of the rat were removed from the bar and the time of removal of one or both paws was recorded. Further, the cut-off time was applied after $120 \mathrm{~s}$.

For the grid test, ${ }^{25}$ rats were hung by its paws on a vertical grid $(25.5 \mathrm{~cm}$ wide and $44 \mathrm{~cm}$ high with spaces of $1 \mathrm{~cm}$ between each wire), and the time for the rats to move its paws or any sort of first movement was recorded as descent latency. The maximum cut off time was fixed at $180 \mathrm{~s}$. 


\subsection{Determination of $\mathrm{VO}_{2}, \mathrm{VCO}_{2}, \mathrm{H}, \mathrm{RER}$ and EE}

The rats in the different groups were individually placed in the respiratory chambers of TSE phenoMaster/LabMaster (TSE phenoMaster/LabMaster, Germany) to monitor $24 \mathrm{~h}$ respiratory parameters under a $12 \mathrm{~h}$ light-dark cycle and it was administered on the 35th day at 8:00 am. Rats in different groups were continuously recorded for $24 \mathrm{~h}$ with the following measurements being taken every $30 \mathrm{~min}$ : water intake, food intake and gas exchange $\left(\mathrm{O}_{2}\right.$ and $\left.\mathrm{CO}_{2}\right)$ (using the TSE LabMaster system). Oxygen consumption $\left(\mathrm{VO}_{2}\right)$ and carbon dioxide production $\left(\mathrm{VCO}_{2}\right)$ were measured and energy expenditure (EE) was calculated according to the manufacturer's guidelines (PhenoMaster Software, TSE Systems). The formula for the calculation of EE was $\mathrm{EE}=\left(3.815+1.232 \times \mathrm{VCO}_{2} / \mathrm{VO}_{2}\right) \times \mathrm{VO}_{2} \times 0.001$. The respiratory exchange rate (RER) was estimated by calculating the ratio of $\mathrm{VCO}_{2} / \mathrm{VO}_{2}$. Values were adjusted by body weight to the power of $0.75\left(\mathrm{~kg}^{-0.75}\right)$ where mentioned. Heat $(\mathrm{H})$ was calculated based on the actual weight of the animal. The room temperature was controlled at $22 \pm 1{ }^{\circ} \mathrm{C}$ in an isolated environment. The sampling flow rate was continuously controlled at $0.25 \mathrm{~L} \mathrm{~min}^{-1}$, with the flow speed set at $1 \mathrm{~L} \mathrm{~min}^{-1}$. This was continuously detected for 24 hours to measure the $\mathrm{VCO}_{2}, \mathrm{VO}_{2}$, H, RER and EE of each group. ${ }^{26}$

\subsection{Tissue preparation for biochemical studies}

At the end point of the experiments, the animals were anesthetized using an intraperitoneal injection of pentobarbital (4 mg per $\mathrm{kg} \mathrm{b}$. wt) and cardiac perfusion was carried out using $0.01 \mathrm{M}$ phosphate-buffered saline (PBS) at $\mathrm{pH} 7.4$ to wash out the blood. The brains of five rats in each group was quickly removed and fixed in $4 \%$ paraformaldehyde (PFA) overnight at $4{ }^{\circ} \mathrm{C}$ and then processed for $\mathrm{TH}, \alpha$-SYN, Iba-1 and GFAP immunoperoxidase staining. The brains of four rats in each group were quickly removed and placed on an ice plate where the two hemispheres were separated carefully. The midbrain region was dissected and immediately frozen in liquid nitrogen for western blot. The brains of four rats in each group were quickly removed and the SN and striatum region were separated carefully and immediately frozen for future use.

\subsection{Biochemical studies}

After behavioral analyses, brain tissues were procured from the control and experimental groups and SN regions were dissected to perform biochemical studies. SN region was homogenized with 9 times the amount of saline and then centrifuged at a speed of $2500 \mathrm{rpm}$ for $10 \mathrm{~min}$. The resulting supernatant was used for the measurement according to correspondent measurement kits. The levels of pro-inflammatory cytokines such as IL-6, IL-1 $\beta$, TNF- $\alpha$, IFN- $\gamma$ and PGE2 in SN region were measured using commercially available ELISA kits and the level of DA in striatum region was measured using DA assay kit. The estimation of markers of oxidative stress such as SOD activity, GSH, GSH-Px activity and MDA used spectrophotometric measurements.

\subsection{Immunofluorescence staining}

Immunofluorescence staining was performed in the $\mathrm{SN}$ to examine the activation of TH, $\alpha$-SYN, GFAP-positive astrocytes and Iba-1-positive microglia. After the brain was fixed overnight in $4 \%$ PFA at $4{ }^{\circ} \mathrm{C}$, it was dehydrated, infiltrated and then embedded into paraffin blocks. The paraffin blocks were cut coronally into $4 \mu \mathrm{m}$-thick sections with a microtome and mounted on glass slide. Slides with brain sections were in an oven at $55{ }^{\circ} \mathrm{C}$ for 2 hours. Then, they were deparaffinized by immersing in xylene two times for $30 \mathrm{~min}$ each and rehydrated for $5 \mathrm{~min}$ each in following solutions, absolute alcohol two times, 95\% alcohol two times, and 85\% alcohol. The slices were added $100 \mu \mathrm{L}$ of $0.1 \%$ trypsin solution, digested at $37{ }^{\circ} \mathrm{C}$ for $10 \mathrm{~min}$ and washed 3 times with PBS for $5 \mathrm{~min}$ each. The slides were blocked with $20 \% \mathrm{H}_{2} \mathrm{O}_{2}$ for $10 \mathrm{~min}$, subsequently washed with PBS twice and non-specific binding with 5\% BSA for $15 \mathrm{~min}$. The sections were then subjected to immunoperoxidase staining using rat monoclonal antibodies overnight (TH, $\alpha$-SYN, GFAP and Iba-1). After staining, the sections were counterstained with hematoxylin, dehydrated twice with absolute alcohol, then cleared in xylene three times for $10 \mathrm{~min}$ each and mounted. The images were taken using light microscope and analyzed using computer image analysis.

\subsection{Western blot}

Protein was extracted from rat SN, and the protein concentration was determined using a total protein assay kit. Samples of protein were mixed with gel loading buffer, boiled for $5 \mathrm{~min}$, separated by $10 \%$ SDS-PAGE, and the proteins were transferred onto a PVDF membrane. Next, membranes were blocked in 5\% BCA for $1 \mathrm{~h}$ and incubated with primary antibodies against rat NF- $\mathrm{B}(1: 1000)$ or COX-2 $(1: 500)$ at $4{ }^{\circ} \mathrm{C}$ overnight and washed with $5 \%$ TBST five times for $5 \mathrm{~min}$ each. Thereafter, membranes were incubated with secondary antibodies for $1 \mathrm{~h}$ and washed with $5 \%$ TBST five times for 5 min each. Immunoreactive bands were visualized using an ECL kit. Relative expression was calculated as the ratio of the gray value of the target protein band to an internal reference band.

\subsection{Statistical analyses}

All data were expressed as the mean \pm standard deviation with the use of SPSS19.0 software. The data were analysed using ANOVA with the one-way LSD test, unless otherwise specified, with Dunnett's T3 analysis. $p$-values of $<0.05$ were considered to be statistically significant.

\section{Results}

\subsection{Behavioral assessment of the effect of arctigenin on rotenone model of $\mathrm{PD}$}

As show in Fig. 1, the deadlock time of ROT rats on wire grid in grid test (A) increased significantly from the second week $\left({ }^{\# \#} p<\right.$ 0.01) compared to the CON group. MDP, MAG and HAG treatment significantly decreased $(* * p<0.01)$ the time on the fourth week, and also the fifth week $(* * p<0.01)$. In bar test $(\mathrm{B})$, from the second week, the deadlock time of ROT rats on wire bar 

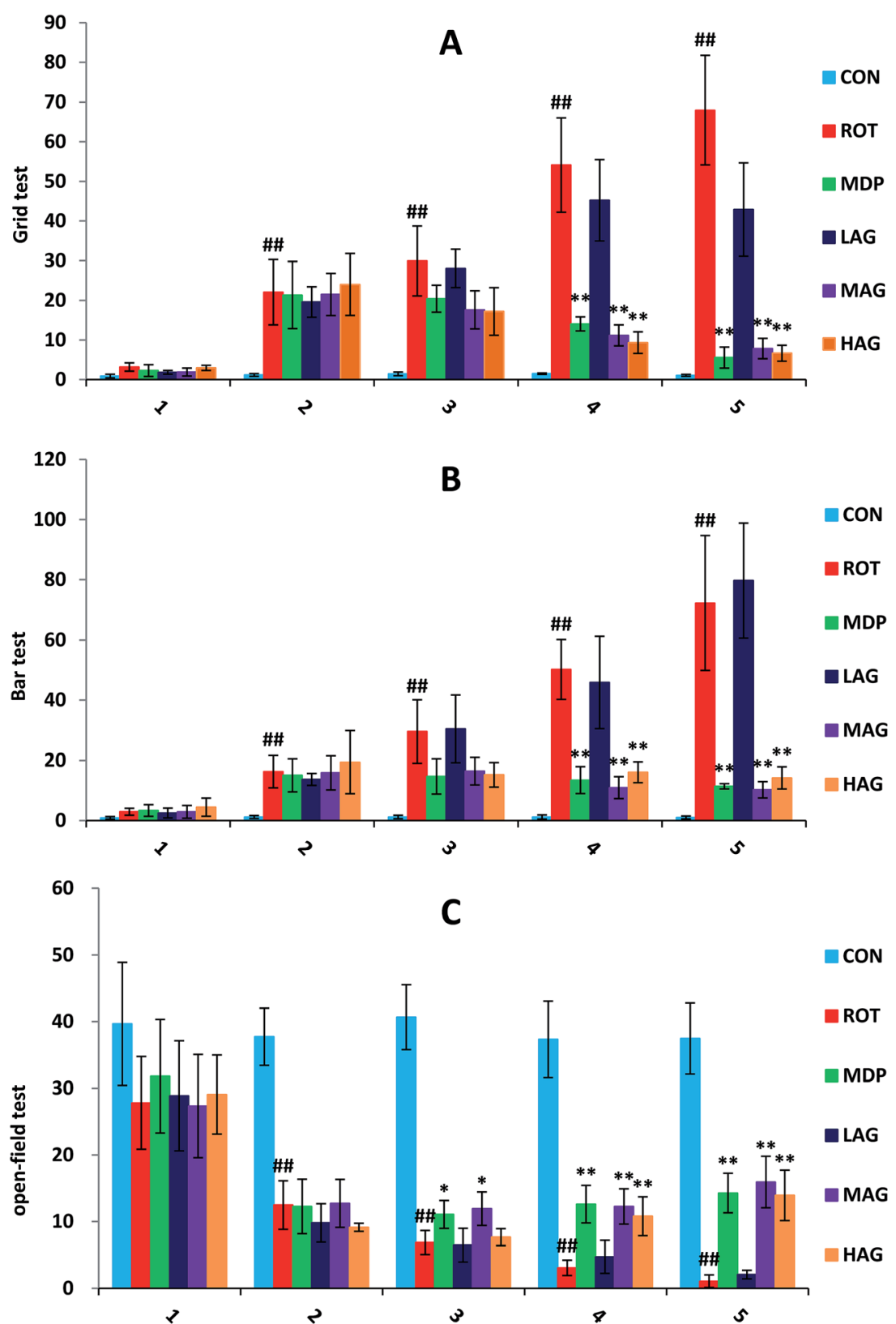

Fig. 1 Effects of arctigenin on behavioral changes induced by rotenone in the behavior test on the 1st, 2nd, 3rd, 4th, 5th weeks. (A) Grid test. (B) Bar test. (C) Open-field test. $n=8$ animals; ${ }^{\# \#} p<0.01$ vs. CON; ${ }^{*} p<0.05, * * p<0.01$ vs. ROT.

increased significantly $\left({ }^{\# \#} p<0.01\right)$ compared to the CON group. MDP, MAG and HAG treatment significantly decreased $(* * p<0.01)$ the time on the fourth week, and also the fifth week $(* * p<0.01)$. In open-field test $(\mathrm{C})$ : from the second week, the distance of ROT rats moved within 5 minutes in the test box decreased significantly ( ${ }^{\# \#} p<0.01$ ) compared to the CON group. MDP, MAG and HAG treatment significantly increased $(* * p<0.01)$ the time on the fourth week, and also the fifth week $(* * p<0.01)$.

\subsection{Effect of arctigenin on energy homeostasis in rat}

As show in Fig. 2, the $\mathrm{VO}_{2}$ in ROT group showed no difference compared with the CON group. However, the $\mathrm{VO}_{2}$ in MAG group was significantly increased compared with the ROT group $(* * p<$ 0.01). Additionally, $\mathrm{VO}_{2}$ in the MDP and HAG group were obviously increased (compared with the ROT group, ${ }^{*} p<0.05$ ). The $\mathrm{VCO}_{2}$ and RER were obviously decreased in the ROT group compared to the CON group $\left({ }^{\# \#} p<0.01\right)$. In contrast, madopar and arctigenin supplementation to the rotenone injected rats remarkably increased the $\mathrm{VCO}_{2}$ and RER in the MDP, MAG and HAG group compared with ROT group $\left({ }^{* *} p<0.01\right)$. The $\mathrm{H}$ and EE were decreased in the ROT group compared to the CON group ( $\left.{ }^{\#} p<0.05\right)$. In contrast, $\mathrm{H}$ and EE in the MDP, MAG and HAG group were obviously increased compared with the ROT group $(* * P<0.01)$. 

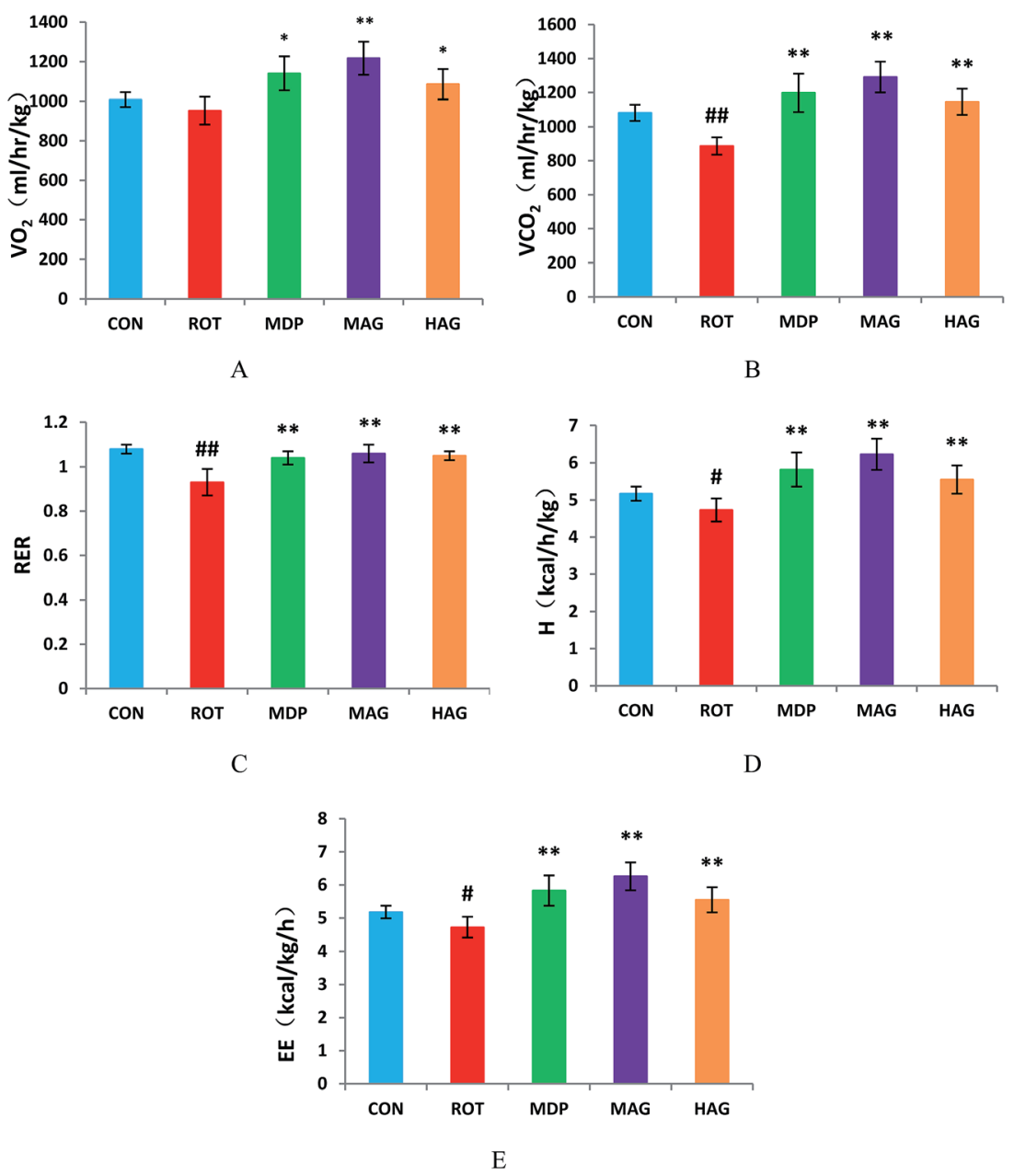

Fig. 2 Effect of arctigenin on energy homeostasis. (A) Oxygen consumption normalized by body weight $\left(\mathrm{VO}_{2}\right)$; (B) carbon dioxide production $\left(\mathrm{VCO}_{2}\right)$; (C) respiratory exchange ratio (RER); (D) heat production $(\mathrm{H})$; (E) energy expenditure (EE). $n=6-7$ animals; ${ }^{\# \#} p<0.01 \mathrm{vs}$. CON; * $p<0.05$, $* * p<0.01$ vs. ROT.

\subsection{Effect of arctigenin on dopaminergic neuronal loss in the SN}

To investigate the beneficial effect of arctigenin on DA neurodegeneration in rotenone-injected rats, TH immunohistochemical analysis was performed to assess the expression of healthy TH positive DA neurons in the SNc. Positive brownish staining for TH was detected in the SNc of the CON group (Fig. 3), while in the ROT group there was a decreased staining of the soma in all animals. Treatment (MDP, MAG and HAG group) led to an increase in the positive brownish staining compared to ROT group. The number of $\mathrm{TH}^{+}$positive DA neurons was decreased $\left({ }^{\#} p<0.01\right)$ in the SN of the ROT group compared to the CON group. MDP, MAG and HAG treatment significantly $\left({ }^{* *} p<0.01\right)$ protected the DA neurons from the rotenone-induced neuronal death. No significant difference was observed in the DA neurons between the ROT and LAG group.

The expression profiles of $\alpha$-synuclein, the key PD-associated proteins, were tested in the rats. As show in Fig. 4, the immunohistochemical results demonstrated that the number of $\alpha$ synuclein immunopositive was increased $\left({ }^{\#} p<0.01\right)$ in the SN of the ROT group compared to the CON group. MDP $\left({ }^{* *} p<0.01\right)$,
MAG $\left({ }^{* *} p<0.01\right)$ and HAG $\left({ }^{*} p<0.05\right)$ treatment significantly decreased the over-expression of $\alpha$-synuclein from the rotenoneinduced neuronal death. No significant difference was observed in expression of $\alpha$-synuclein between the ROT and LAG group.

\subsection{Effect of arctigenin on oxidative stress in the SN}

As show in Fig. 5, rotenone exposure induced a significant increase ( $\left.{ }^{\#} p<0.01\right)$ in the MDA level (A) in the SN of the ROT group compared to the CON group. MAG $\left({ }^{* *} p<0.01\right)$, HAG $\left({ }^{*} p<\right.$ $0.05)$ and $\operatorname{MDP}\left({ }^{* *} p<0.01\right)$ supplementation to the rotenoneadministered rats significantly decreased the level of MDA. No significant difference was observed between the ROT and LAG group. GSH, SOD, GSH-Px: rotenone injection also significantly decreased in the GSH level $\left({ }^{\#} p<0.05\right)$ (B) and the activity of SOD $\left({ }^{\# \#} p<0.01\right)$ (C) and GSH-Px $\left({ }^{\# \#} p<0.01\right)$ (D) compared to the CON group. MAG arctigenin supplementation significantly inhibited the rotenone-induced decrease in the GSH level $\left({ }^{*} p<0.05\right)$ and the activities of SOD $(* p<0.05)$ and GSH-Px $(* * p<0.01)$ compared to the ROT group. HAG arctigenin supplementation significantly inhibited the rotenone-induced decrease $\left({ }^{* *} p<\right.$ 0.01 ) in SOD and GSH-Px activity compared to the ROT group. 

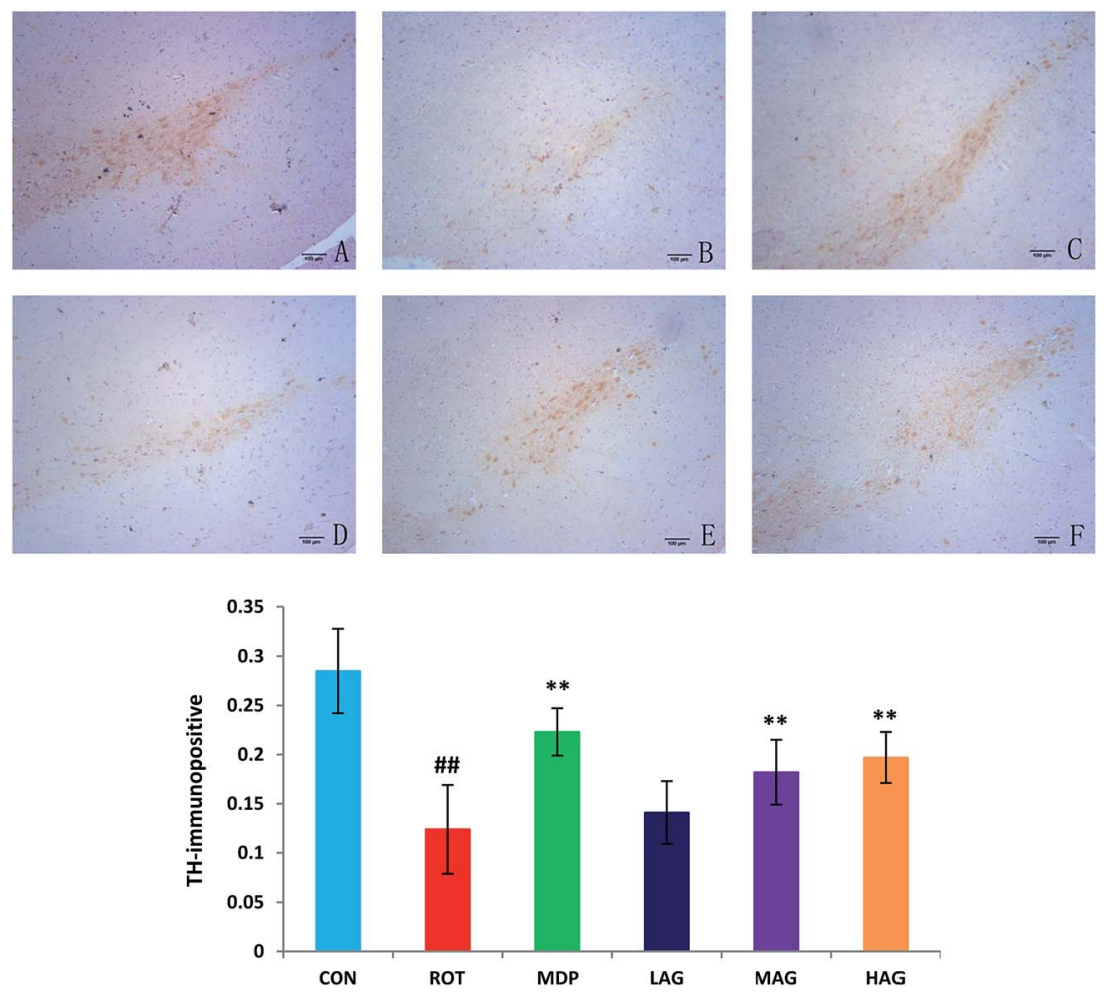

Fig. 3 Effect of arctigenin on TH-immunopositive $\left(\mathrm{TH}^{+}\right)$DA neurons in the SN. (A) CON; (B) ROT; (C) MDP; (D) LAG; (E) MAG; (F) HAG. $n=4$ animals; ${ }^{\# \#} p<0.01$ vs. CON; $* * p<0.01$ vs. ROT.

MDP supplementation just increase in SOD $(* * p<0.01)$ compared to the ROT group. No significant difference was observed between the ROT and LAG group.

\subsection{Effect of arctigenin on proinflammatory cytokines in the SN}

The concentrations of pro-inflammatory cytokines, IL-6, IL-1 $\beta$, TNF- $\alpha$, IFN- $\gamma$, PGE2 and NO, in the SN were also measured to determine the effect of arctigenin on neuroinflammation induced by rotenone as show in Fig. 6 . A significant increase in SN of IL-6, IL-1 $\beta$, TNF- $\alpha$, IFN- $\gamma$ and PGE2 levels $\left({ }^{\# \#} p<0.01\right)$ and NO level ( $\left.{ }^{\# \#} p<0.01\right)$ was observed in ROT group as compared to CON group. Arctigenin administration in MAG group resulted in a significant decrease in SN of IL-6, IL- $1 \beta$, TNF- $\alpha$, IFN- $\gamma$, NO levels $(* * p<0.01)$ and PGE2 level $(* p<0.05)$ in arctigenintreated rats in comparison to rotenone exposed group. Arctigenin treatment in HAG group significantly decreased the rotenone-induced increase of IL- 6 , TNF- $\alpha$, IFN- $\gamma$, PGE2 levels $\left({ }^{* *} p<0.01\right)$ and IL-1 $\beta$ and NO levels $\left({ }^{*} p<0.05\right)$ compared to the ROT group. However, madopar treatment just decreased levels of IFN- $\gamma$, PGE2 $(* p<0.05)$ and TNF- $\alpha(* * p<0.01)$. Treatment with arctigenin in LAG group did not show any significant change in the levels of these proinflammatory cytokines.

\subsection{Effect of arctigenin on the induction of glial cells activation in the $\mathrm{SN}$}

Immunofluorescence staining of GFAP and Iba- 1 were carried out to determine the morphological changes in astrocytes and microglia, respectively as show in Fig. 7 and 8. High expressions of GFAP (Fig. 8) and Iba-1 (Fig. 7) were observed in rotenoneinjected rats when compared to $\mathrm{CON}$ rats that indicate increased numbers of astrocytes and microgia with astrocyte hypertrophy and microglial activation. However, arctigenin treatment of ROT-injected rats showed a comparatively decreased immunofluorescence reactivity of GFAP and Iba-1. The number of GFAP and Iba-1-positive astrocytes and microglia in the rats of different experimental groups was also counted. The number of Iba-1 immunopositive was increased in the SN of the ROT group compared to the CON group ( $\left.{ }^{\# \#} p<0.01\right)$ in the number of activated microglia. In contrast, MAG $\left({ }^{* *} p<0.01\right)$ and HAG $\left({ }^{*} p<\right.$ 0.05) supplementation to the rotenone-injected rats showed moderate staining of Iba- 1 compared to the ROT group in the number of activated microglia. No significant difference was observed in expression of Iba- 1 between the ROT and LAG group. The number of GFAP immunopositive was increased in the SN of the ROT group compared to the CON group $\left({ }^{\# \#} p<0.01\right)$ in the number of activated astrocytes. In contrast, MAG $(* * p<0.01)$, HAG $(* p<0.01)$ and MDP $(* * p<0.05)$ supplementation to the rotenone injected rats showed moderate staining of GFAP compared to the ROT group in the number of activated astrocytes $(* * p<0.01)$. No significant difference was observed in expression of GFAP between the ROT and LAG group.

\subsection{Effect of arctigenin on inflammatory mediators expressions of NF- $\kappa \mathrm{B}$ and $\mathrm{COX}-2$}

Expressions of NF- $\kappa \mathrm{B}$ and COX-2 using western blot in the SN tissue were also determined as show in Fig. 9. The ROT group 

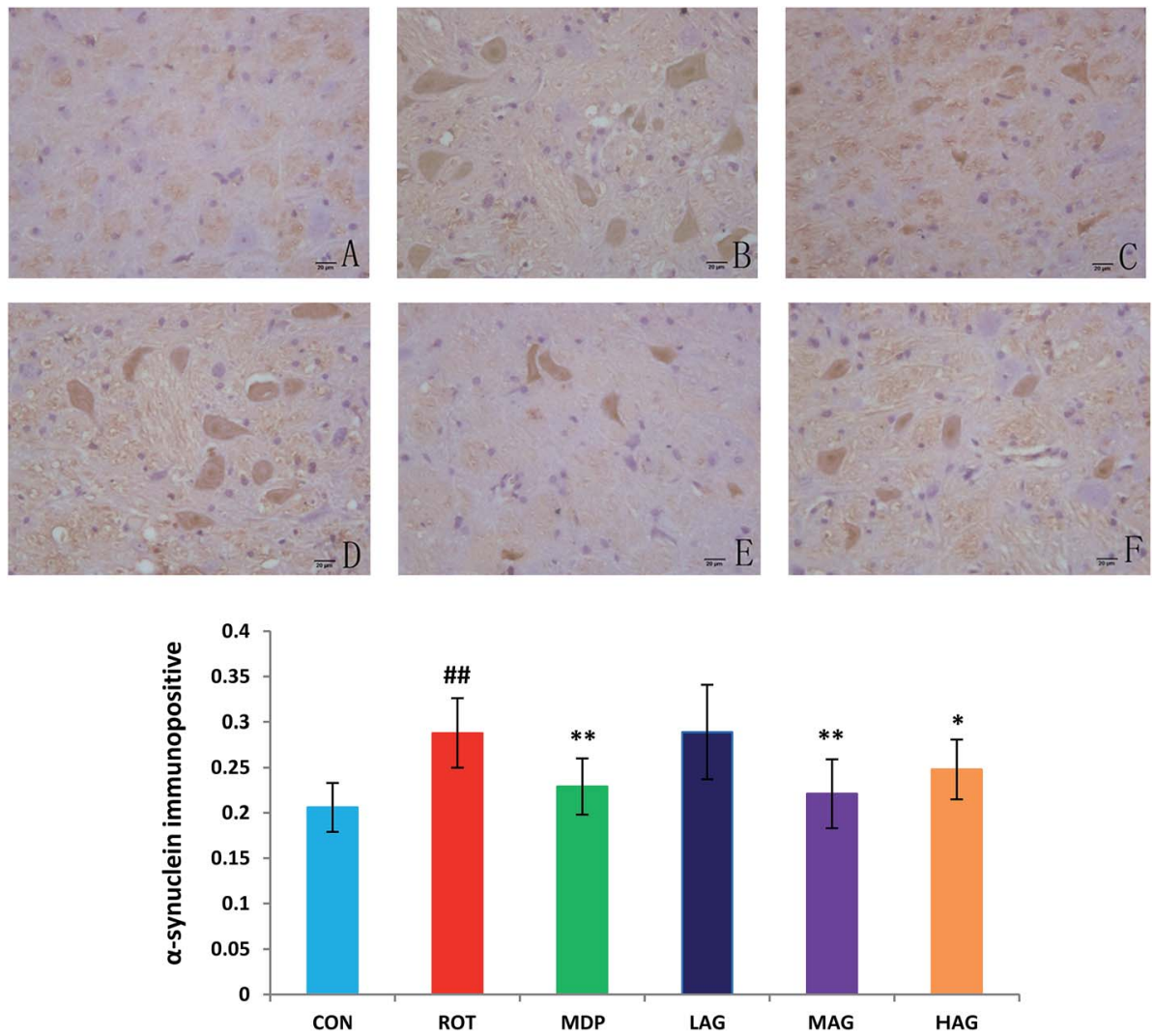

Fig. 4 Effect of arctigenin on $\alpha$-synuclein-immunopositive $\left(\alpha\right.$-synuclein $\left.{ }^{+}\right)$in the SN. (A) CON; (B) ROT; (C) MDP; (D) LAG; (E) MAG; (F) HAG. $n=4$ animals; ${ }^{\# \#} p<0.01$ vs. CON; ${ }^{*} p<0.05,{ }^{* *} p<0.01$ vs. ROT.

showed significant increase $\left({ }^{\# \#} p<0.01\right)$ in the NF-кB level compared to the CON group. MAG, HAG and MDP supplementation to the rotenone-injected rats remarkably decreased (**p $<0.01)$ the expression level of NF- $\kappa \mathrm{B}$ compared to the ROT group (A). No significant differences were observed between the ROT and LAG group. Likewise, COX-2 expression was also
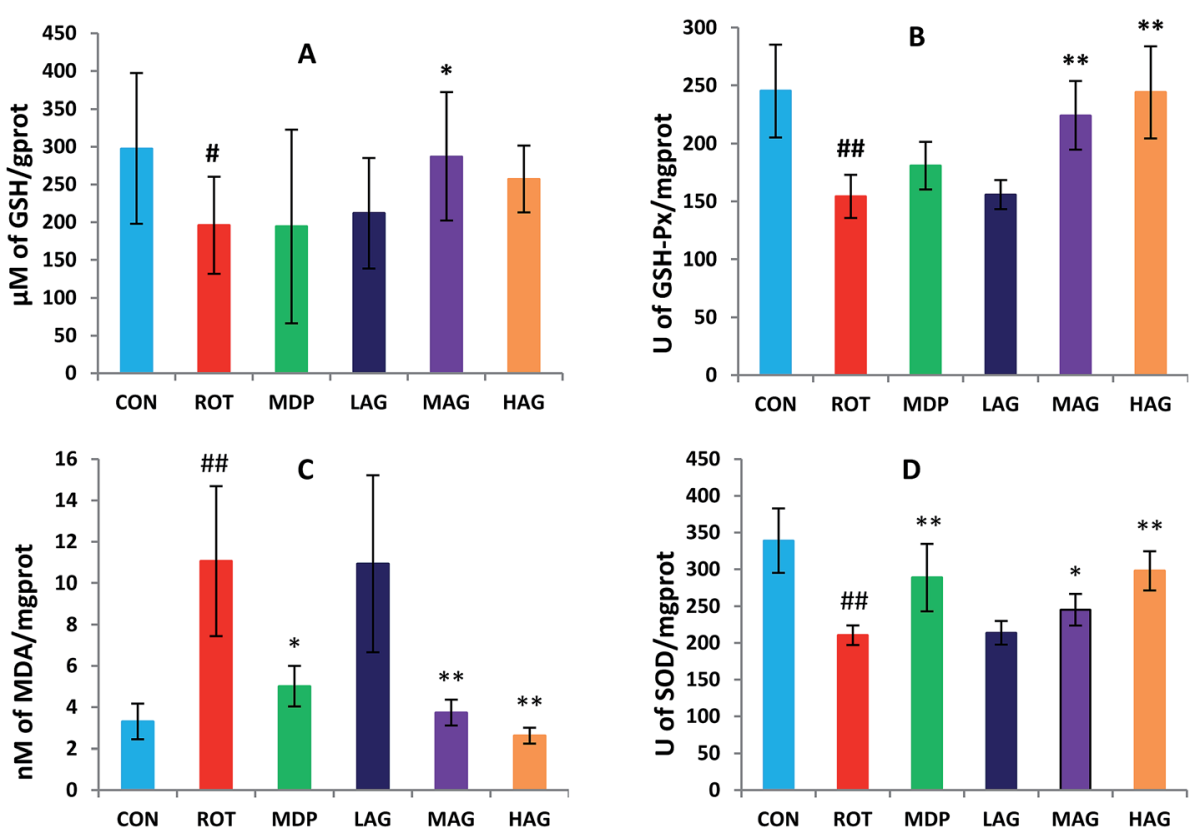

Fig. 5 Effect of arctigenin on oxidative stress in the SN. (A) MDA; (B) GSH; (C) SOD; (D) GSH-Px. $n=7-9$ animals; ${ }^{\# \#} p<0.01$ vs. CON; *p $<0.05$, $* * p<0.01$ vs. ROT 

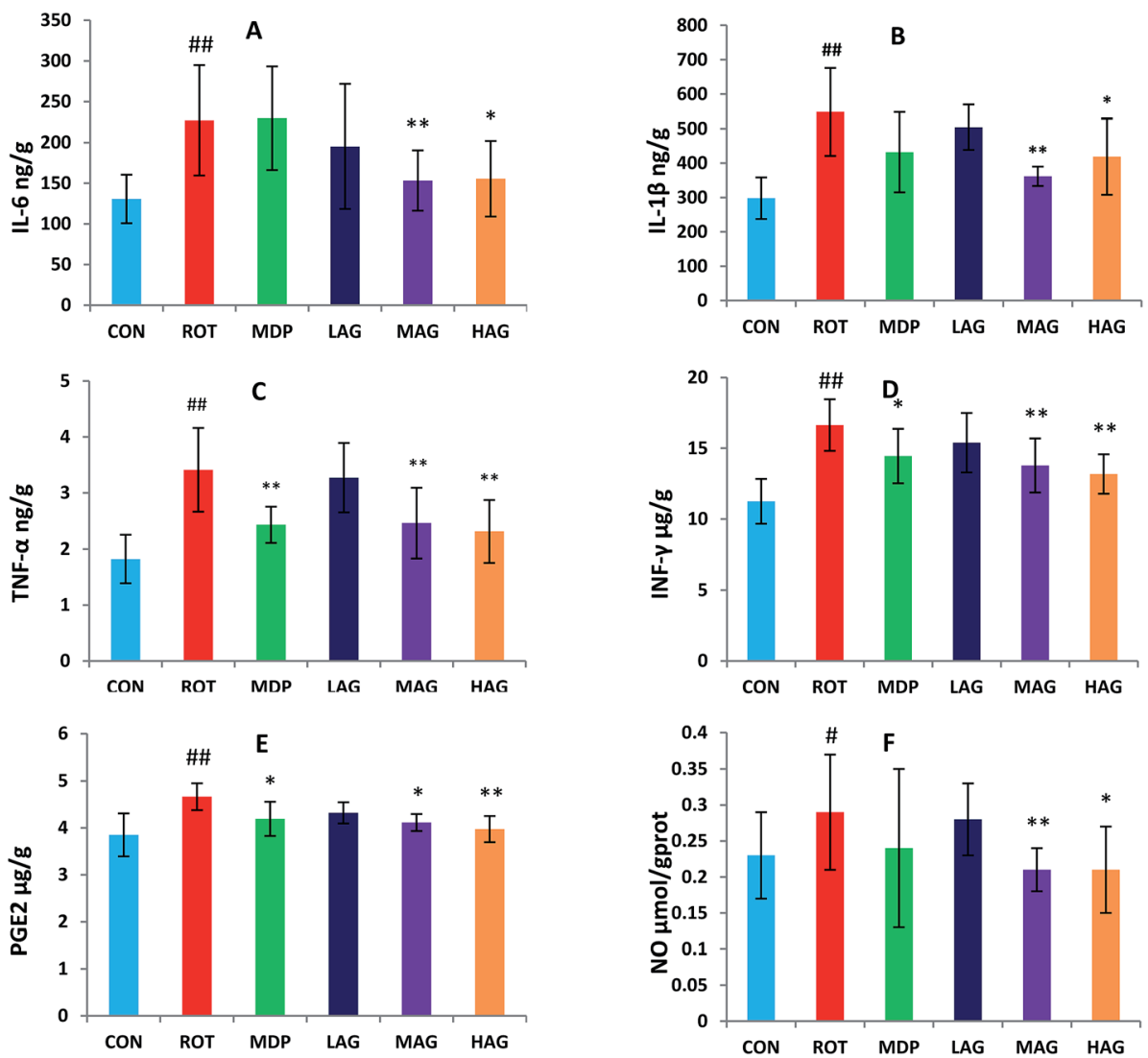

Fig. 6 Effect of arctigenin on proinflammatory cytokines in the SN. (A) IL-6; (B) IL-1 1 ; (C) TNF- $\alpha$; (D) IFN- $\gamma ;$ (E) PGE2; (F) NO. $n=7-9$ animals; \#\# $p$ $<0.01$ vs. CON; $* p<0.05, * * p<0.01$ vs. ROT.

significantly increased $\left({ }^{\#} p<0.01\right)$ in the ROT group compared to the CON group. MAG $\left({ }^{* *} p<0.01\right)$, HAG $\left({ }^{*} p<0.05\right)$ and MDP $\left({ }^{* *} p<0.01\right)$ treatment markedly decreased the COX-2 expression compared to the ROT group (B). No significant differences were observed between the ROT and LAG group.

\section{Discussion}

$\mathrm{PD}$ is one of the most common progressive neurodegenerative disorders with a complex pathogenesis. Arctigenin, a naturally lignan compound, has been reported to be protective against neurodegenerative disease such as Alzheimer's disease. ${ }^{23}$ However, the effect of arctigenin against PD is not known. In this study, we have aimed to evaluate the neuroprotective effects of arctigenin against rotenone-induced dopaminergic neuronal toxicity in PD rats. It has been reported in the literature that arctigenin is non-toxic orally in rats. ${ }^{27}$ The results of preliminary experiments showed that $60 \mathrm{mg} \mathrm{kg}{ }^{-1}$ was effective dose in rats. Therefore, we set up $20 \mathrm{mg} \mathrm{kg}^{-1}$ for low dose, $60 \mathrm{mg} \mathrm{kg}^{-1}$ for medium dose and $150 \mathrm{mg} \mathrm{kg}^{-1}$ for high dose in this experiment. To our knowledge, this is the first report of effect of arctigenin in PD rats. Rotenone is a pesticide which induces PD-like behavioral and pathological symptoms through multiple mechanisms, such as dopaminergic neurodegeneration, $\alpha$-synucleinopathy, mitochondrial complex-I inhibition, oxidative stress, inflammation, and glial activation. ${ }^{28-31}$ In the current study, the rotenone-induced rat model was undertaken to investigate the neuroprotective effect of arctigenin. Rotenone at the dose of $2.0 \mathrm{mg} \mathrm{kg}^{-1}$ once daily for 5 weeks was used to induce the dopaminergic neurodegeneration as reported in a previous study. ${ }^{32}$ The results of the study show the novel potential benefits of arctigenin in attenuating motor impairments and dopaminergic neurodegeneration, improving energy metabolism, and reducing oxidative stress and inflammatory in the brain. Therefore, anti-inflammatory and antioxidant agents could be an effective therapeutic strategy in PD by regulating oxidative stress and subsequent neuroinflammation, dopaminergic degeneration, and motor abnormalities.,33,34

In this study, the rat model of PD induced by rotenone was used to observe the therapeutic effect of arctigenin on PD. From the results of grid experiment, open field experiment and open field experiment, the middle dose $\left(60 \mathrm{mg} \mathrm{kg}^{-1}\right)$ and high dose (150 $\mathrm{mg} \mathrm{kg}^{-1}$ ) of arctigenin and positive drug Madopa can significantly improve the general condition and motor function of PD model rats, indicating that arctiagenin has a therapeutic effect on rotenone-induced PD rats. PD lesions are mainly due to degeneration of DA neurons in the SN and DA deficiency in the striatum. ${ }^{35} \mathrm{TH}$ is the key rate-limiting enzyme for tyrosine conversion to DA. Increasing the content of $\mathrm{TH}$ protein and increasing the release of DA into the striatum of the $\mathrm{SN}$ is one of the strategies for the treatment of PD. In this study, the number of TH-positive cells in the SN of the ROT group was significantly 

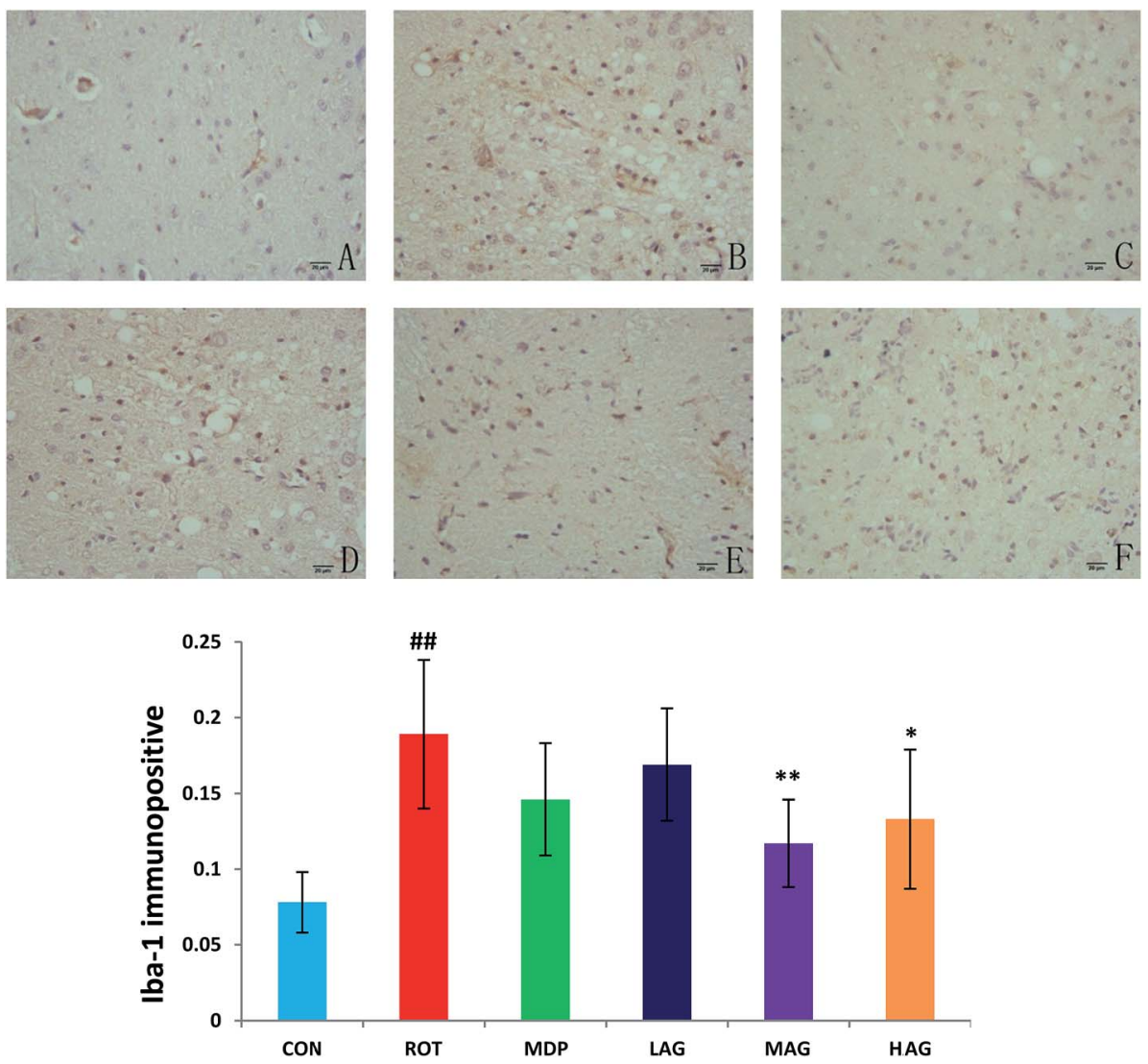

Fig. 7 Effect of arctigenin on Iba-1-immunopositive (Iba-1 ${ }^{+}$) in the SN. (A) CON; (B) ROT; (C) MDP; (D) LAG; (E) MAG; (F) HAG. $n=4$ animals; \#\# $p$ $<0.01$ vs. CON; $* p<0.05, * * p<0.01$ vs. ROT.

lower than that in the control group. At the same time, the expression of TH-positive cells in the SN of the arctiagenin group was significantly higher than that in the model group. This is a histological proof of the success of the rat model of PD disease, also shows that arctiagenin can reduce the rotenonecaused damage on PD rats in the SN dopaminergic neurons. Behavioral changes in rats statistical results with histology together to make a proof.

$\alpha$-SYN is the most important protein factor in the pathogenesis of PD. It is one of the main components of the characteristic pathological marker of PD. In recent years, more and more studies have shown that $\alpha$-SYN abnormal aggregation is closely related to the pathogenesis of PD. ${ }^{36}$ Lehmensiek et al. ${ }^{37}$ found that overexpression or abnormal aggregation of $\alpha$-SYN can lead to mitochondrial dysfunction, promote mitochondrial susceptibility to oxidative stress, dopamine transportermediated toxicity and cell death. Abnormal aggregation of $\alpha$ SYN can lead to oxidative stress and inflammation in neuronal cells, and make them interact with each other to promote the pathogenesis of PD. In this study, $\alpha$-SYN immunohistochemical results showed that the positive expression of $\alpha$-SYN in the SN of the model group was significantly higher than that in the control group. At the same time, the expression of $\alpha$-SYN in the $\mathrm{SN}$ of the arctigenin rats was significantly lower than that in the model group. Although there is no effective way to prevent $\alpha$ SYN pathological aggregation, but if you can make $\alpha$-SYN abnormal aggregation reduce or accelerate the removal of $\alpha$ SYN clearance, it is possible to use this as the basis for drug treatment. Arctigenin treatment may reduce the accumulation of $\alpha$-SYN in the SN by anti-oxidation and anti-inflammatory, and have a positive effect on the maintenance and behavior of dopaminergic neurons in the SN of the PD model rats.

According to the statistics of the ventilator, $\mathrm{VCO}_{2}, \mathrm{VO}_{2}, \mathrm{H}$, RER and EE in the PD model group were significantly lower than those in the control group. It was found that the respiratory metabolism of PD rats was decreased. It is presumed that the $\mathrm{VCO}_{2}, \mathrm{VO}_{2}, \mathrm{H}, \mathrm{RER}$ and EE in PD rats were lower than those in normal rats because of the decrease of muscle rigidity and autonomic activity in PD rats. The results of behavioral experiments showed that the number of crossing grids and standing times of PD rats was significantly decreased, and the autonomy activity was significantly reduced, which was consistent with the experimental results. The $\mathrm{VCO}_{2}, \mathrm{VO}_{2}, \mathrm{H}, \mathrm{RER}$ and $\mathrm{EE}$ of Madopa group, middle dose arctiagenin group and high dose of arctiagenin group were significantly higher than those of model group after treatment with Madopa and arctigenin. The results showed that the metabolic rate of PD rats was adjusted to normal rats after treatment with Madopa and arctigenin, which could significantly improve the proactivity of PD rats.

Oxidative stress and free radical damage are the key aspects of PD mechanism. In the normal physiological state of the body, mitochondria will produce reactive oxygen species (ROS), and 

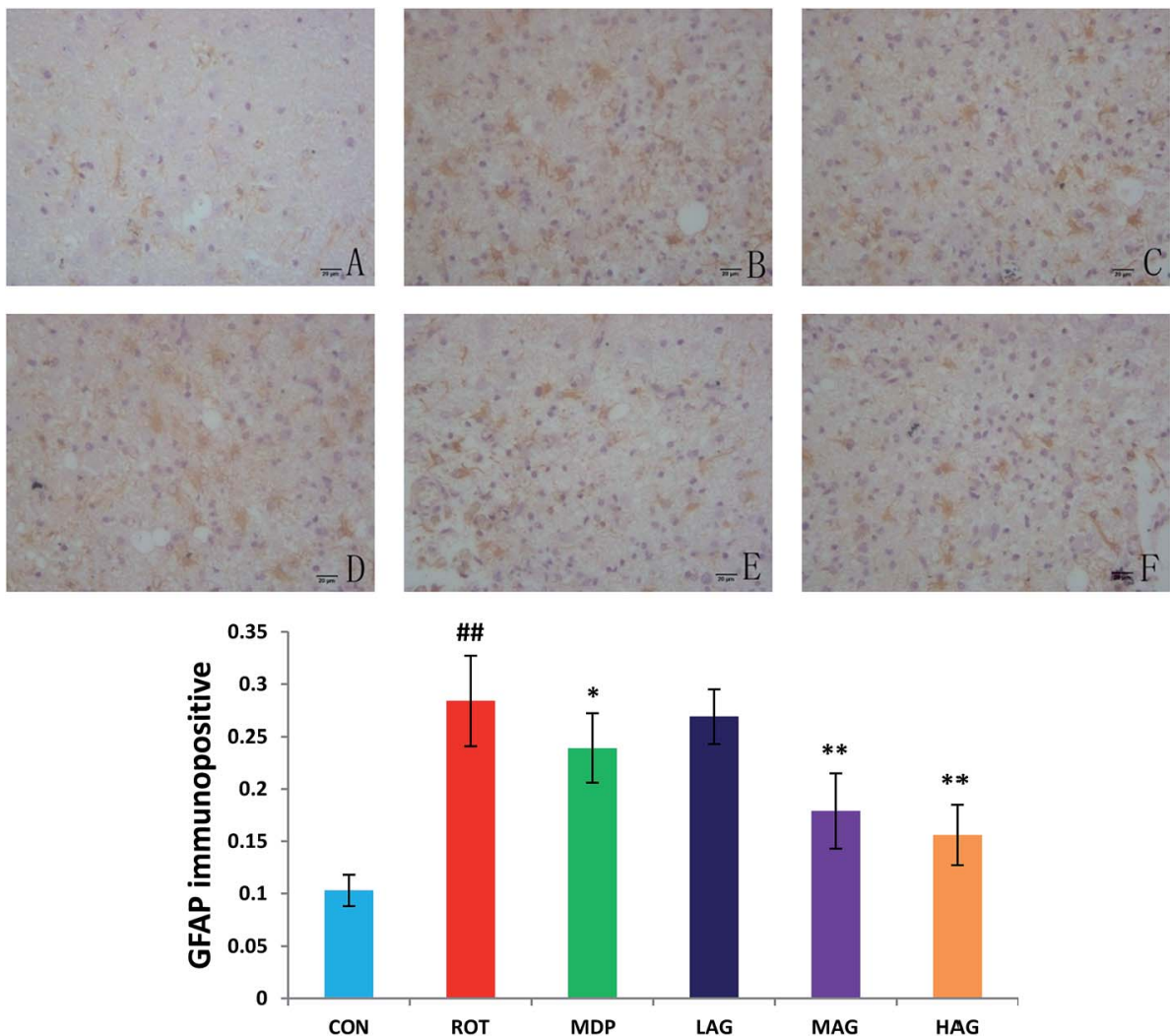

Fig. 8 Effect of arctigenin on GFAP-immunopositive (GFAP ${ }^{+}$) in the SN. (A) CON; (B) ROT; (C) MDP; (D) LAG; (E) MAG; (F) HAG. $n=4$ animals; \#\# $p$ $<0.01$ vs. CON; $* p<0.05, * * p<0.01$ vs. ROT.

these superoxide compounds will be reduced by superoxide dismutase. The generation and removal of free radicals in the body are in equilibrium. However, many compounds inhibit the activity of the respiratory chain and block the electron transport chain. When this balance is broken, excessive accumulation of free radicals in the nerve cells leads to oxidative stress. DA metabolism in DA neurons also produces ROS, which increase the likelihood of oxidative damage. ${ }^{38}$ In addition, the central nervous system is rich in lipid components, oxygen consumption is relatively high, and there is a lack of antioxidant enzymes in the central nervous system, so it is particularly sensitive to free radical damage. ${ }^{39}$ Therefore, excessive ROS damage to the
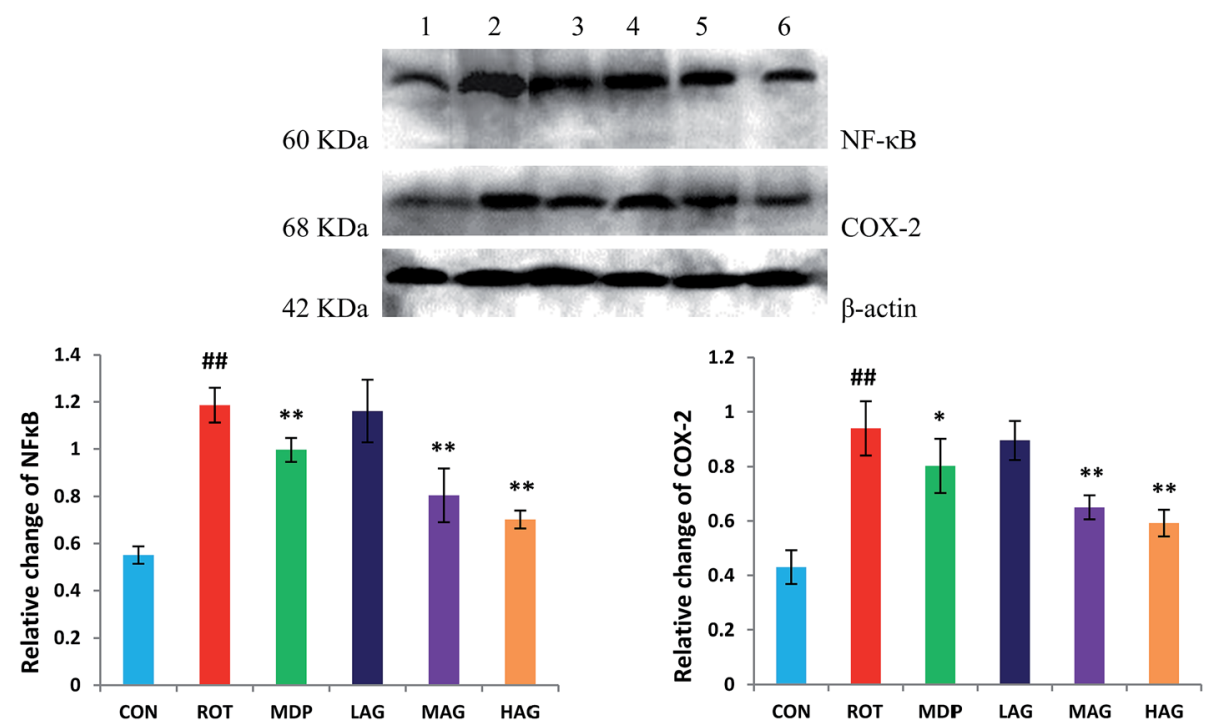

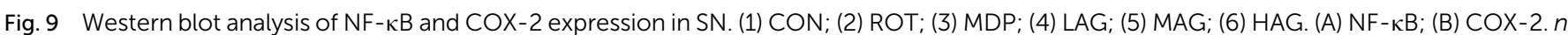
$=3$ animals; ${ }^{\# \#} p<0.01$ vs. CON; ${ }^{*} p<0.05,{ }^{* *} p<0.01$ vs. ROT. 
mitochondria and other biological macromolecules, resulting in a vicious cycle of oxidative stress that eventually leads to apoptosis of nerve cells. ${ }^{40}$ In addition, oxidative stress can also cause aggregation of $\alpha$-SYN in the cytoplasm of human brain DA neurons. ${ }^{41} \alpha$-SYN that has not been mutated has been damaged by oxygen free radicals, which can be deposited in cells, leading to neurotoxicity. In the pathogenesis of $\mathrm{PD}$, mitochondrial function loss and oxidative stress can interact with each other, and the relationship is very close. The free radicals produced by oxidative stress can inhibit complex I, while the suppression of complex I can produce more free radicals and produce oxidative stress. The combined effect of oxidative stress and mitochondrial dysfunction can lead to apoptosis of nerve cells, resulting in PD. ${ }^{38,42} \mathrm{GSH}$ is an important non-enzymatic antioxidant in the body, which can reduce the peroxidation ability of free radical by reducing of hydrogen peroxide. GSH is necessary for GSH-Px to decompose hydroperoxide, so the amount of GSH is an important factor in measuring the body's antioxidant capacity. The depletion of cellular GSH levels has been reported in dopaminergic areas of the PD brain to overcome escalated oxidative stress following ROT challenge. ${ }^{43}$ SOD can remove oxygen free radicals by disproportionation, and protect against oxidative damage. Free radicals can attack biofilm to induce lipid peroxidation, resulting in lipid peroxidation products such as MDA. The weakening of antioxidant defenses such as GSH, SOD, and GSH-Px has been well documented in the PD brain. ${ }^{2}$ The experimental results showed that administration of rotenone caused significant reduction in the activities of SOD and GSH-Px as well as reduced GSH levels that demonstrate the occurrence of oxidative stress in the rat brain. However, compared with ROT group, arctigenin treatment could significantly decrease the content of MDA in lipid peroxidation product, restoring the content of GSH and the activities of antioxidant enzymes, SOD and GSH-Px. The decrease in the MDA content along with restoration of GSH and activities of SOD and GSH-Px by arctigenin is linked with its free radical scavenging and antioxidant activity, consistent with the reports in the literature. ${ }^{\mathbf{4 4 - 4 6}}$ This result may be due to the existence of a phenolic hydroxyl group and three methoxyls in the structure of the arctigenin, and the methoxyl group has taken off the methyl group in the animal, resulting in more phenolic hydroxyl group, which are reductive.

In addition to oxidative stress, the activation of microglia in the brain is common and an early event in the onset of neurodegeneration contributing to several neuropathologies. ${ }^{47}$ Microglia and astrocytes can be activated in a variety of ways during the development and progression of $\mathrm{PD}$, and participate in the initiation and amplification of neuronal injury, resulting in a large number of free radicals, superoxide anions, TNF- $\alpha$, IL$1 \beta$, NO, PGE2 and other substances that subsequently cause the dopaminergic neurodegeneration..$^{48}$ Activation of glial cells was observed following chronic rotenone injections as evidenced by increased expression of Iba-1 and GFAP which are considered to be indicators of microglial and astrocyte activation, respectively, and subsequent neuroinflammation which is in agreement with previous reports. ${ }^{49-51}$ In the present study, the expression of Iba1 and GFAP in the SN of the model group was significantly higher than that in the control group, and the microglia and astrocytes were over-activated. The treatment of medium and high dose of arctigenin has a significant improvement in the role, which can partially inhibit the activation of microglial and astrocyte, suggesting that it may inhibit the activation of microglial and astrocyte or reduce inflammation caused by microglial and astrocyte activation to reduce neuronal damage.

The central inflammatory process, which includes the activation of microglia and secretion of pro-inflammatory cytokines, are key players in the neurodegenerative process of PD. ${ }^{52}$ In order to investigate the anti-inflammatory ability of the arctigenin to rotenone-induced neuroinflammation of $\mathrm{PD}$, the levels of pro-inflammatory cytokines and inflammatory mediators were investigated. The experimental results showed that the levels of IL- 6 , IL-1 $\beta$, TNF- $\alpha$, IFN- $\gamma$, PGE2 and NO in the SN of the model group induced with rotenone were significantly increased compared with those in the control group similar to numerous previous reports. ${ }^{38,49}$ The expression of $\mathrm{NF}-\kappa \mathrm{B}$ and COX-2 increased in the $\mathrm{SN}$ of rats administrated with rotenone in the present study. It can be seen that the microglia and astrocytes in the $\mathrm{SN}$ of rats administrated with rotenone are activated and many cytokines, such as IL-6, IL-1 $\beta$, TNF- $\alpha$, IFN- $\gamma$ etc., are significantly upregulated by activated microglia, which affect the surrounding astrocytes and neurons. Activated microglia cause phosphorylation and degradation of the inhibitory factor $\mathrm{I} \kappa \mathrm{B}$ which results in the activation of the NF- $\kappa \mathrm{B}$ signaling cascade. ${ }^{53} \mathrm{NF}-\kappa \mathrm{B}$ is activated into the nucleus and binds to specific sites on DNA to regulate expression of IL-1 $\beta$, IL6 , TNF- $\alpha$, iNOS and COX-2 and other genes. ${ }^{54,55}$ TNF- $\alpha$ is mainly produced by microglia and astrocytes, which can induce the upregulation of MHC I molecules in neurons, making them susceptible to attack and lead to destruction of neurons in the central nervous system. TNF- $\alpha$ can also induce astrocytes to release NO, arachidonic acid and glutamate and other neurotoxic substances, expanding the inflammatory response. IFN- $\gamma$ is secreted by microglia in inflammation, which in turn IFN- $\gamma$ can activate microglia, induce up-regulation of MHC II, promote iNOS transcription, increase NO production, and form positive feedback to increase neurons toxic damage. Inflammatory cytokines interact with each other, forming a waterfall effect and leading to a vicious circle. In addition, the inflammatory reaction leads to the transcription of iNOS and the mass production of NO. INOS can produce a large amount of NO and superoxide radicals, which can cause serious oxidative damage directly or indirectly. ${ }^{53}$ Thus, a variety of reasons cause the activation of microglia and then release the proinflammatory cytokines, start or amplify the damage to neurons, and the neuronal damage and proinflammatory cytokines in turn activate microglia. Inflammation and oxidative stress interact and promote each other, leading to neuronal cell damage and death. Neuronal damage can also make more of the microglia activation, thus further damage to neighboring neurons, forming a vicious cycle of neuron degeneration necrosis constantly, ${ }^{56}$ in agreement with previous reports. However, arctigenin plays an important role in controlling the levels of IL-1 $\beta$, IL- 6 , IFN- $\gamma$ and TNF- $\alpha$ by counteracting microglial activation. In addition, treatment with arctigenin leads to the attenuation of NF- $\kappa \mathrm{B}$ and 
COX-2 levels. In this study, down-regulation of NF- $\kappa \mathrm{B}$ and COX2 along with attenuation of pro-inflammatory cytokines can be ascribed to the multimodal anti-inflammatory properties of arctigenin in conferring protection against rotenone-induced dopaminergic neuronal loss. The findings from the present study and previous reports suggest arctigenin to be a promising agent of natural origin to protect against PD and neurodegeneration.

\section{Conclusion}

The findings demonstrated that arctigenin can improve the behavior changes of PD rats and the damage of DA neurons. The oxidative stress and inflammation are involved in the pathogenesis of PD and arctigenin may protect DA neurons through its potent antioxidant and anti-inflammatory activities.

\section{Conflicts of interest}

There are no conflicts to declare.

\section{References}

1 A. Gopalakrishna and S. A. Alexander, Understanding Parkinson Disease: A Complex and Multifaceted Illness, Journal of Neuroscience Nursing, 2015, 47(6), 320-326.

$2 \mathrm{R}$. Niranjan, The role of inflammatory and oxidative stress mechanisms in the pathogenesis of Parkinson's disease: focus on astrocytes, Mol. Neurobiol., 2014, 49(1), 28-38.

3 J. M. Taylor, B. S. Main and P. J. Crack, Neuroinflammation and oxidative stress: co-conspirators in the pathology of Parkinson's disease, Neurochem. Int., 2013, 62(5), 803-819.

4 M. Murai and H. Miyoshi, Current topics on inhibitors of respiratory complex I, Biochim. Biophys. Acta, 2016, 1857(7), 884-891.

5 N. Xiong, X. Long, J. Xiong, et al., Mitochondrial complex I inhibitor rotenone-induced toxicity and its potential mechanisms in Parkinson's disease models, Crit. Rev. Toxicol., 2012, 42(7), 613-632.

6 L. H. Sanders and J. T. Greenamyre, Oxidative damage to macromolecules in human Parkinson disease and the rotenone model, Free Radical Biol. Med., 2013, 62, 111-120.

7 T. B. Sherer, J. H. Kim, R. Betarbet, et al., Subcutaneous rotenone exposure causes highly selective dopaminergic degeneration and alpha-synuclein aggregation, Exp. Neurol., 2003, 179(1), 9-16.

8 T. B. Sherer, R. Betarbet, J. H. Kim, et al., Selective microglial activation in the rat rotenone model of Parkinson's disease, Neurosci. Lett., 2003, 341(2), 87-90.

9 F. Blandini and M. T. Armentero, Animal models of Parkinson's disease, FEBS J., 2012, 279(7), 1156-1166.

10 J. R. Cannon and J. T. Greenamyre, The role of environmental exposures in neurodegeneration and neurodegenerative diseases, Toxicol. Sci., 2011, 124(2), 225-250.

11 M. E. Johnson and L. Bobrovskaya, An update on the rotenone models of Parkinson's disease: their ability to reproduce the features of clinical disease and model gene- environment interactions, Neurotoxicology, 2015, 46, 101116.

12 A. O. Pires, F. G. Teixeira, B. Mendes-Pinheiro, et al., Old and new challenges in Parkinson's disease therapeutics, Prog. Neurobiol., 2017, 156, 69-89.

13 R. Ceravolo, C. Rossi, E. Del Prete, et al., A review of adverse events linked to dopamine agonists in the treatment of Parkinson's disease, Expert Opin. Drug Saf., 2016, 15(2), 181-198.

14 H. Javed, S. Azimullah, K. S. Abul, et al., Neuroprotective effect of nerolidol against neuroinflammation and oxidative stress induced by rotenone, BMC Neurosci., 2016, 17(1), 58.

15 S. Ojha, H. Javed, S. Azimullah, et al., $\beta$-Caryophyllene, a phytocannabinoid attenuates oxidative stress, neuroinflammation, glial activation, and salvages dopaminergic neurons in a rat model of Parkinson disease, Mol. Cell. Biochem., 2016, 418(1-2), 59-70.

16 K. Tamilselvam, N. Braidy, T. Manivasagam, et al., Neuroprotective Effects of Hesperidin, a Plant Flavanone, on Rotenone-Induced Oxidative Stress and Apoptosis in a Cellular Model for Parkinson's Disease, Oxid. Med. Cell. Longevity, 2013, 2013, 1-11.

17 G. Zhang, N. Xiong, Z. Zhang, et al., Effectiveness of traditional Chinese medicine as an adjunct therapy for Parkinson's disease: a systematic review and meta-analysis, PLoS One, 2015, 10(3), e118498.

18 J. Y. Cho, A. R. Kim, E. S. Yoo, et al., Immunomodulatory effect of arctigenin, a lignan compound, on tumour necrosis factor-alpha and nitric oxide production, and lymphocyte proliferation, J. Pharm. Pharmacol., 1999, 51(11), 1267-1273.

19 M. K. Cho, J. W. Park, Y. P. Jang, et al., Potent inhibition of lipopolysaccharide-inducible nitric oxide synthase expression by dibenzylbutyrolactone lignans through inhibition of I-kappaBalpha phosphorylation and of p65 nuclear translocation in macrophages, Int. Immunopharmacol., 2002, 2(1), 105-116.

20 M. K. Cho, Y. P. Jang, Y. C. Kim, et al., Arctigenin, a phenylpropanoid dibenzylbutyrolactone lignan, inhibits MAP kinases and AP-1 activation via potent MKK inhibition: the role in TNF-alpha inhibition, Int. Immunopharmacol., 2004, 4(10-11), 1419-1429.

21 S. R. Hyam, I. A. Lee, W. Gu, et al., Arctigenin ameliorates inflammation in vitro and in vivo by inhibiting the PI3K/ AKT pathway and polarizing M1 macrophages to M2-like macrophages, Eur. J. Pharmacol., 2013, 708(1-3), 21-29.

22 F. S. Predes, A. L. Ruiz, J. E. Carvalho, et al., Antioxidative and in vitro antiproliferative activity of Arctium lappa root extracts, BMC Complementary Altern. Med., 2011, 11, 25.

23 Z. Zhu, J. Yan, W. Jiang, et al., Arctigenin effectively ameliorates memory impairment in Alzheimer's disease model mice targeting both beta-amyloid production and clearance, J. Neurosci., 2013, 33(32), 13138-13149.

24 S. Chompoopong, S. Jarungjitaree, T. Punbanlaem, et al., Neuroprotective Effects of Germinated Brown Rice in Rotenone-Induced Parkinson's-Like Disease Rats, NeuroMol. Med., 2016, 18(3), 334-346. 
25 A. M. Teema, S. A. Zaitone and Y. M. Moustafa, Ibuprofen or piroxicam protects nigral neurons and delays the development of l-dopa induced dyskinesia in rats with experimental Parkinsonism: Influence on angiogenesis, Neuropharmacology, 2016, 107, 432-450.

26 S. M. Hofmann, L. Zhou, D. Perez-Tilve, et al., Adipocyte LDL receptor-related protein-1 expression modulates postprandial lipid transport and glucose homeostasis in mice, J. Clin. Invest., 2007, 117(11), 3271-3282.

27 H. E. Li, B. Li, J. C. Yao, et al., Study on 28-day Repeated Oral Dose Toxicity of Arctigenin in Rats, Chinese Journal of Pharmacovigilance, 2016, 13(6), 325-329.

28 J. X. Song, S. C. Sze, T. B. Ng, et al., Anti-Parkinsonian drug discovery from herbal medicines: what have we got from neurotoxic models?, J. Ethnopharmacol., 2012, 139(3), 698711.

29 J. R. Cannon, V. Tapias, H. M. Na, et al., A highly reproducible rotenone model of Parkinson's disease, Neurobiol. Dis., 2009, 34(2), 279-290.

30 T. B. Sherer, R. Betarbet, J. H. Kim, et al., Selective microglial activation in the rat rotenone model of Parkinson's disease, Neurosci. Lett., 2003, 341(2), 87-90.

31 S. Ojha, H. Javed, S. Azimullah, et al., Neuroprotective potential of ferulic acid in the rotenone model of Parkinson's disease, Drug Des., Dev. Ther., 2015, 9, 54995510.

32 S. Ojha, H. Javed, S. Azimullah, et al., Neuroprotective potential of ferulic acid in the rotenone model of Parkinson's disease, Drug Des., Dev. Ther., 2015, 9, 5499-5510.

33 J. J. Sutachan, Z. Casas, S. L. Albarracin, et al., Cellular and molecular mechanisms of antioxidants in Parkinson's disease, Nutr. Neurosci., 2012, 15(3), 120-126.

34 S. L. Albarracin, B. Stab, Z. Casas, et al., Effects of natural antioxidants in neurodegenerative disease, Nutr. Neurosci., 2012, 15(1), 1-9.

35 M. Messripour and A. Mesripour, Age related interaction of dopamine and serotonin synthesis in striatal synaptosomes, Biocell, 2013, 37(2), 17-21.

36 K. S. Mcnaught and C. W. Olanow, Protein aggregation in the pathogenesis of familial and sporadic Parkinson's disease, Neurobiol. Aging, 2006, 27(4), 530-545.

37 V. Lehmensiek, E. M. Tan, J. Schwarz, et al., Expression of mutant alpha-synucleins enhances dopamine transportermediated MPP+ toxicity in vitro, NeuroReport, 2002, 13(10), 1279-1283.

38 P. Thakur and B. Nehru, Anti-inflammatory properties rather than anti-oxidant capability is the major mechanism of neuroprotection by sodium salicylate in a chronic rotenone model of Parkinson's disease, Neuroscience, 2013, 231, 420431.

39 J. Davis, S. Moylan, B. H. Harvey, et al., Neuroprogression in schizophrenia: pathways underpinning clinical staging and therapeutic corollaries, Aust. New Zeal. J. Psychiatr., 2014, 48(6), 512-529.

40 S. Pizzimenti, E. Ciamporcero, M. Daga, et al., Interaction of aldehydes derived from lipid peroxidation and membrane proteins, Front. Physiol., 2013, 4, 242.
41 M. S. Wang, S. Boddapati, S. Emadi, et al., Curcumin reduces alpha-synuclein induced cytotoxicity in Parkinson's disease cell model, BMC Neurosci., 2010, 11, 57.

42 L. C. Assis, M. R. Straliotto, D. Engel, et al., betaCaryophyllene protects the C6 glioma cells against glutamate-induced excitotoxicity through the Nrf2 pathway, Neuroscience, 2014, 279, 220-231.

43 M. Smeyne and R. J. Smeyne, Glutathione metabolism and Parkinson's disease, Free Radicals Biol. Med., 2013, 62, 13-25.

44 A. Li, X. Zhang, M. Shu, et al., Arctigenin suppresses renal interstitial fibrosis in a rat model of obstructive nephropathy, Phytomedicine, 2017, 30, 28-41.

45 S. Su and M. Wink, Natural lignans from Arctium lappa as antiaging agents in Caenorhabditis elegans, Phytochemistry, 2015, 117, 340-350.

46 W. Z. Zhang, Z. K. Jiang, B. X. He, et al., Arctigenin Protects Against Lipopolysaccharide-Induced Pulmonary Oxidative Stress and Inflammation in a Mouse Model via Suppression of MAPK, HO-1, and iNOS Signaling, Inflammation, 2015, 38(4), 1406-1414.

47 K. J. Doorn, P. J. Lucassen, H. W. Boddeke, et al., Emerging roles of microglial activation and non-motor symptoms in Parkinson's disease, Prog. Neurobiol., 2012, 98(2), 222-238.

48 R. L. Mosley, E. J. Benner, I. Kadiu, et al., Neuroinflammation, Oxidative Stress and the Pathogenesis of Parkinson's Disease, Clin. Neurosci. Res., 2006, 6(5), 261281.

49 S. Ojha, H. Javed, S. Azimullah, et al., Neuroprotective potential of ferulic acid in the rotenone model of Parkinson's disease, Drug Des., Dev. Ther., 2015, 9, 54995510.

50 S. Sarkar, B. Gough, J. Raymick, et al., Histopathological and electrophysiological indices of rotenone-evoked dopaminergic toxicity: neuroprotective effects of acetyl-Lcarnitine, Neurosci. Lett., 2015, 606, 53-59.

51 S. Swarnkar, P. Goswami, P. K. Kamat, et al., Rotenoneinduced neurotoxicity in rat brain areas: a study on neuronal and neuronal supportive cells, Neuroscience, 2013, 230, 172-183.

52 P. L. Mcgeer and E. G. Mcgeer, Glial reactions in Parkinson's disease, Mov. Disord., 2008, 23(4), 474-483.

53 D. Litteljohn, E. Mangano, M. Clarke, et al., Inflammatory mechanisms of neurodegeneration in toxin-based models of Parkinson's disease, J. Parkinson's Dis., 2010, 2011, 713517.

54 K. Guo, X. Mou, J. Huang, et al., Trans-caryophyllene suppresses hypoxia-induced neuroinflammatory responses by inhibiting NF-kappaB activation in microglia, J. Mol. Neurosci., 2014, 54(1), 41-48.

55 Y. Cheng, Z. Dong and S. Liu, beta-Caryophyllene ameliorates the Alzheimer-like phenotype in APP/PS1 mice through CB2 receptor activation and the PPARgamma pathway, Pharmacology, 2014, 94(1-2), 1-12.

56 M. L. Block and J. S. Hong, Microglia and inflammationmediated neurodegeneration: multiple triggers with a common mechanism, Prog. Neurobiol., 2005, 76(2), 77-98. 